\title{
Inhalt des Jahrgangs 35/Sommaire du volume 35
}

Erwin H.Ackerknecht, Gudden, Huguenin, Hitzig. Hirnpsychiatrie im Burghölzli 1869-1879

Erwin H.Ackerknecht, Johann Georg Zimmermann (1728-1795) 224

Heinz Balmer, Fritz Fischer $\quad 107$

Jost Benedum, Der Badearzt Asklepiades und seine bithynische Heimat 20

René Bernoulli, Descartes' Grundgedanken in medizinhistorischer Sicht 44.

Heinrich Buess, Der Neuenburger Arzt Louis Guillaume (1833-1924), ein fast vergessener Pionier des staatlichen Gesundheitsdienstes 230

Esther Fischer-Homberger, Zwerchfellverletzung und psychische Störung 1

Stephanos Geroulanos, Iakovos Pylarinos (1659-1718) und sein Beitrag zur Variolation 264.

Wolfgang Gresky, Zwei Briefe des Berner Professors Johann Georg Tralles an Georg Christoph Lichtenberg (1786)

Walter Kaiser, Die zeitliche Ausbreitung von Potentialen in der Elektrodynamik $\quad 297$

Andreas Kleinert und Charlotte Schönbeck, Lenard und Einstein. Ihr Briefwechsel $\begin{array}{ll}\text { und ihr Verhältnis vor der Nauheimer Diskussion von } 1920 & 318\end{array}$

Fridolf Kudlien, Zwei Interpretationen zum Hippokratischen Eid 253

Zdenko Levental, Josef Koetschet - ein Schweizer in ärztlichen und diplomatischen Diensten der Türkei

Cléopâtre Montandon, Naissance des relations entre justice et psychiatrie à Genève

Heinrich Rodegra, Mary Lindeman und Martin Ewald, Kindermord und verheimlichte Schwangerschaft in Hamburg im 18. Jahrhundert

Hans-Konrad Schmutz, Barocke und klassizistische Elemente in der anatomischen Abbildung

Rudolf Steiger, Conrad Geßners Itinerar seiner Bündner Reise von 1561

Clemens Stoll, Das Preßburger Arznei-Schuldbuch (1578-1584)

\section{Kürzere Beiträge}

Urs Boschung, Kolloquium zur Schweizer Pestgeschichte

\section{Halleriana:}

Paul Röthlisberger, Eine Reminiszenz an die Bibliothek A.v. Hallers

Heinz Balmer, Der Gedenkstein für Hallers jüngste Tochter. - Albrecht von Haller der Jüngere (1758-1823)

\section{Nachrufe}

Frau Emilie Guggenheim-Schnurr. 7. Mai 1906 bis 26. Juni 1978 (Huldrych M. Koelbing) 341 


\section{Buchbesprechungen}

José de Acosta, Historia natural y moral de las Indias (Erwin H. Ackerknecht)

Albucasis, On Surgery and Instruments (Huldrych M. Koelbing)

Jean-Noël Biraben, Les hommes et la peste en France et dans les pays européens et méditerranéens (Huldrych M. Koelbing und Vera Waldis)

Wilhelm Blasius, Krankheit und Heilung im Märchen (Heinz Balmer)

Arthur L. Donovan, Philosophical Chemistry in the Scottish Enlightenment. The Doctrines and Discoveries of William Cullen and Joseph Black (Jean Starobinski)

Wolfgang Eckart und Klaus-Peter Pohl, Das Studium der Medizin und die Fächer «Theorie der Medizin» und «Geschichte der Medizin» im Urteil der Medizinstudenten (Huldrych M. Koelbing)

Markus Fierz, Girolamo Cardano (1501-1576), Arzt, Naturphilosoph, Mathematiker, Astronom und Traumdeuter (Antoinette Stettler)

Werner Fischel, Grundzüge des Zentralnervensystems des Menschen, 4. Auflage (Huldrych M. Koelbing)

Paul Gasser, Charles Krafft (1863-1921), ein Pionier der Appendektomie und der Krankenpflege in Europa (Stephanos Geroulanos)

Roy O. Greep, Marjorie A.Koblinsky, Frederick S.Jaffé (Hrsg.), Reproduction and Human Welfare: A Challenge to Research (Antoinette Stettler)

Hermann Grensemann, Knidische Medizin, Teil 1 (Huldrych M. Koelbing)

Dominique Haefeli-Till, Der «Liber de oculis» des Constantinus Africanus (René Bernoulli)

Albrecht von Haller, 1708-1777. Zehn Vorträge (Heinz Balmer)

Albrecht von Hallers Briefe an Auguste Tissot 1754-1777. Herausgegeben von

Erich Hintzsche (Erna Lesky)

Albrecht Hirschmüller, Physiologie und Psychoanalyse in Leben und Werk Josef Breuers

(Hans H. Walser)

Die Hirsebreifahrt der Zürcher nach Straßburg, 1576 (Paul Röthlisberger)

Prim. Dr. Martin Horvat (1910-1972) (Zdenko Levental)

Emmy Klieneberger-Nobel, Pionierleistungen für die Medizinische Mikrobiologie.

Lebenserinnerungen (Heinz Balmer)

Huldrych M. Koelbing, Arzt und Patient in der antiken Welt (Erna Lesky) 149

Erna Lesky, The Vienna Medical School of the 19th Century (Erwin H.Ackerknecht) 153

Walter Müri, Griechische Studien. Ausgewählte wort- und sachgeschichtliche Forschungen zur Antike (Huldrych M. Koelbing)

Die Rechtsquellen des Kantons Bern. Erster Teil. Bd. X. Bearbeitet von Hermann Rennefahrt (Paul Röthlisberger)

Bento P. M. Schulte und Lambertus J. Endtz, A Short History of Neurology in the Netherlands (Erwin H. Ackerknecht)

Peter Sommer, Das Jenner-Kinderspital in Bern 1862-1962. Fritz Leu, Die Jennerstiftung 1963-1977 (Paul Röthlisberger)

Heinrich Staehelin, Geschichte des Kantons Aargau 1830-1885 (Vera Waldis)

Florian Tennstedt, Geschichte der Selbstverwaltung in der Krankenversicherung 